\title{
Masas mediastinales: Epidemiología y decisiones estratégicas. Experiencia de 13 años
}

\author{
Tulio Torres-Rodríguez,凶 Danilo Herrera-Cruz, Mario Gálvez-González, Edgar Moran-Ocaña, \\ Rosa María del Cid-Herrera, Roberto Gordillo-Castillo
}

Hospital San Vicente, Guatemala.

Trabajo recibido: 13-VIII-2016; aceptado: 8-IX-2016

\begin{abstract}
RESUMEN. Introducción: El mediastino, localizado en el centro del tórax contiene órganos y tejidos de vital importancia y requiere de gestos quirúrgicos precisos para la solución de aquello que le concierne. El objetivo del estudio es presentar la epidemiología de las masas mediastinales y las diferentes modalidades de tratamiento indicado de acuerdo con el diagnóstico del paciente. Diseño, lugar y participantes: Se admitieron todos los pacientes mayores de 13 años con diagnóstico de masa mediastinal evaluados durante el período de noviembre de 2002 a octubre de 2015. Se incluyeron variables demográficas, localización anatómica, diagnóstico clínico y patológico y modalidades de tratamiento quirúrgico. Resultados: Se intervino un total de 844 pacientes con patología quirúrgica torácica, de los cuales 65 (7.7\%) tuvieron diagnóstico de masa mediastinal. Los diagnósticos más frecuentes fueron: tumores de células germinales, 15.38\%; linfomas, 13.84\%; Schwannoma, $12.30 \%$; y tumores del timo, $10.76 \%$. El compartimento mediastínico más afectado fue el anterosuperior en el $63 \%$ de los casos y la toracotomía exploratoria, en la que se incluyen todos los procedimientos de resección, fue el procedimiento mayormente efectuado en el 50.76\%. Conclusiones: La patología mediastinal representó el 7\% de los procedimientos quirúrgicos torácicos realizados. El mediastino anterosuperior fue el compartimento más afectado prevaleciendo el diagnóstico de teratoma, la toracotomía exploratoria con resección de la masa predominó sobre las otras opciones quirúrgicas.
\end{abstract}

Palabras claves: Mediastino, masas, tratamiento.

ABSTRACT. Introduction: The mediastinum, located in the center of the chest contains organs and tissues of vital importance and it requires precise surgical gestures for the solution of what is concerned. The objective of the study is to present the epidemiology of the Mediastinal Masses and different modalities of treatment indicated according to the diagnosis of the patient. Design, place and participants: All patients over the age of 13 with a diagnosis of mass mediastinal evaluated during the period from November 2002 to October 2015 were admitted. Demographic variables, anatomic location, clinical and pathological diagnosis and surgical treatment modalities were included. Results: A total of 844 patients with thoracic surgical pathology was operated of which $65(7.7 \%)$ patients had diagnosis of mediastinal mass; being the most frequent diagnoses, the cells germinal tumors $15.38 \%$, lymphomas $13.84 \%$, Schwannoma $12.30 \%$ and tumors of the thymus gland $10.76 \%$. Most affected mediastinal compartment was the anterosuperior in $63 \%$ of cases and exploratory thoracotomy, which includes all procedures of resection, was the procedure carried out mostly in the $50.76 \%$. Conclusions: Mediastinal pathology represented $7 \%$ of the thoracic surgical procedures performed. The anterosuperior mediastinum was the most affected compartment prevailing diagnosis of teratoma and exploratory thoracotomy with resection of the mass prevailed over other surgical options.

Key words: Mediastinal, masses, treatment.

\section{INTRODUCCIÓN}

El mediastino es la región anatómica ubicada entre el esternón y la columna vertebral en el sentido anteroposterior, las pleuras lateralmente, el opérculo torácico por arriba y el diafragma por debajo, ${ }^{1}$ contiene muchos órganos y tejidos que en la ruptura del equilibrio de la salud-enfermedad pueden ser susceptibles de padecer patologías que deben ser tratadas. Se divide en tres compartimentos: mediastino anterosuperior entre el ángulo de Louis y la cuarta vértebra torácica; mediastino medio entre el pericardio y la porción anterior de la tráquea; mediastino posterior, localizado posterior del pericardio fibroso y al diafragma y anterior a los cuerpos vertebrales de las ocho vértebras dorsales inferiores. Las masas mediastinales del compartimento anterior son las más frecuentes y representan el 50\% de los tumores mediastinales. ${ }^{2}$ La división anatómica por compartimentos propuesto por Shields ${ }^{3,4}$ permite ubicar las diferentes patologías y facilitar la toma precisa 
de decisiones. El objetivo de este estudio es presentar nuestra experiencia sobre la epidemiología de las masas mediastinales y las decisiones estratégicas de tratamiento tomadas de acuerdo con el diagnóstico de los pacientes durante los últimos 13 años.

\section{MÉTODO}

Tras la aprobación por el Comité de Ética se realizó una revisión de todos los pacientes mayores de 13 años de edad que consultaron o fueron ingresados al Departamento de Cirugía General y Torácica del Hospital San Vicente, Guatemala, o a una unidad hospitalaria privada con impresión clínica de masa mediastinal durante el período de noviembre de 2002 a octubre de 2015. Se incluyen variables demográficas, localización anatómica, diagnóstico clínico y patológico, además de las diferentes modalidades de tratamiento.

\section{RESULTADOS}

De noviembre de 2002 a octubre de 2015 se realizaron 844 procedimientos de cirugía torácica, de los cuales a $65(7.7 \%)$ pacientes se les diagnosticó masa mediastinal. El 60\% (39) correspondieron al sexo masculino y $40 \%$ (26) al sexo femenino. La edad tuvo una distribución bimodal, encontrando mayoría de casos en las décadas de 21 a 30 años (20\%) y de 51 a 60 (26\%) (figura 1).

En relación con la presentación clínica, el síntoma predominante fue tos, como motivo de consulta único o acompañado de dolor y/o disnea, y un alto porcentaje fue hallazgo incidental en estudios por sintomatología pulmonar (tabla 1).

El mediastino anterior fue el compartimento más afectado con 41 casos (63\%), el mediastino medio con $10(15 \%)$ y el mediastino posterior con $14(22 \%)$.
Entre las patologías más frecuentes encontramos: tumores de células germinales con 10 casos (15.38\%) predominando los teratomas (figura 2), linfomas en 9 (13.84\%), Schwannoma en 8 (12.30\%), tumores del timo en $7(10.76 \%$ ) (figura 3 ) y carcinoma neuroendocrino en 3 (4.61\%). Las modalidades terapéuticas efectuadas implicaron principalmente: toracotomía exploratoria posterolateral con resección de las masas en 33 pacientes (50.76\%), mediastinostomía en 10 (15.38\%), videotoracoscopia (VATS) en $7(10.76 \%)$ y biopsia ganglionar en 5 (7.69\%) (tabla 2).

Al analizar los resultados de acuerdo con la división anatómica por compartimentos encontramos el mediastino anterior con 41 pacientes; los diagnósticos más frecuentes fueron: linfoma en 8 (19.51\%), teratoma $7(17.03 \%)$ y timoma $6(14.63 \%)$. Los procedimientos realizados fueron: toracotomía exploratoria en 14 pacientes (34.14\%), seguida de mediastinostomía en $10(24.39 \%)$ y la VATS en $6(14.63 \%)$.

De los 10 pacientes con patología del mediastino medio, el quiste pericárdico fue encontrado en $2(20 \%)$ y la toracotomía exploratoria fue el procedimiento mayormente realizado (50\%).

Finalmente en el mediastino posterior, el Schwannoma fue el tumor más frecuente en 8 pacientes (57.14\%). Se incluyó una paciente con pólipo gigante fibrovascular del esófago (figura 4A y 4B). La toracotomía exploratoria fue el procedimiento quirúrgico empleado en el $100 \%$ de los casos (tabla 3).

\section{DISCUSIÓN}

La evaluación de las masas mediastinales por la complejidad de las estructuras del mediastino y la variedad de sus patologías debe ser multidisciplinaria. La distribución por compartimentos ${ }^{3}$ permite la identificación de los diagnósticos diferenciales más probables de

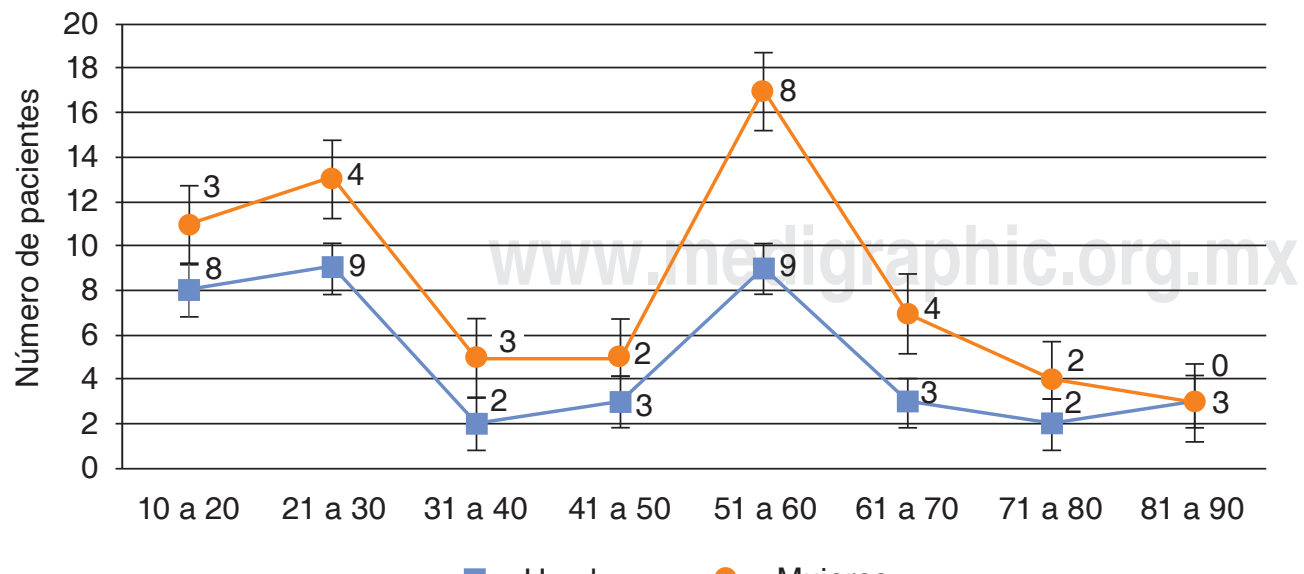

$\longrightarrow$ Hombres $\longrightarrow$ Mujeres
Figura 1.

Relación edad y sexo de los pacientes. 
Tabla 1. Presentación clínica.

\begin{tabular}{lcc}
\hline Síntomas & Número & Porcentaje \\
\hline Tos y dolor & 10 & 15.38 \\
Tos y disnea & 8 & 12.30 \\
Tos & 11 & 16.92 \\
Dolor & 6 & 9.23 \\
Hallazgo incidental & 24 & 36.92 \\
Otros & 6 & 9.23 \\
Total & 65 & 99.97 \\
\hline
\end{tabular}

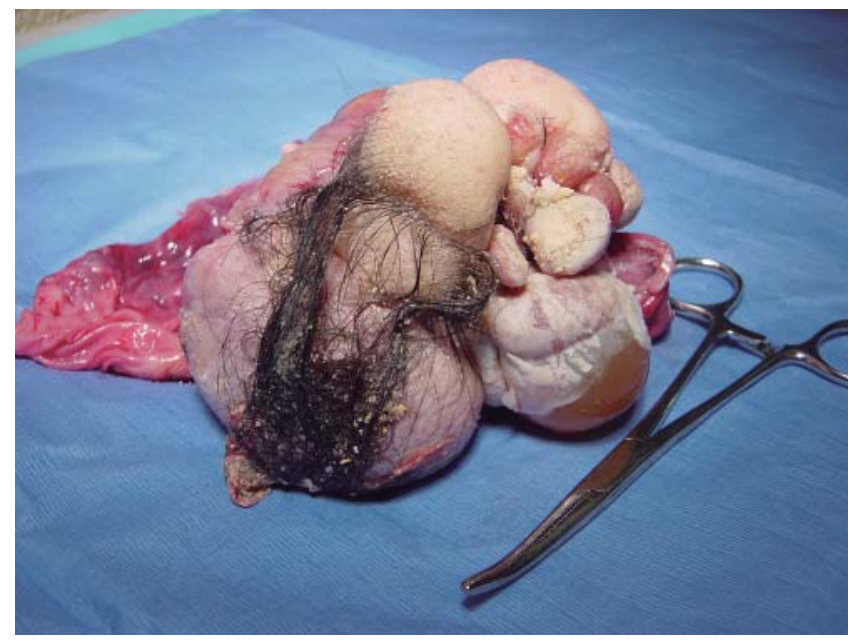

Figura 2. Teratoma maduro.

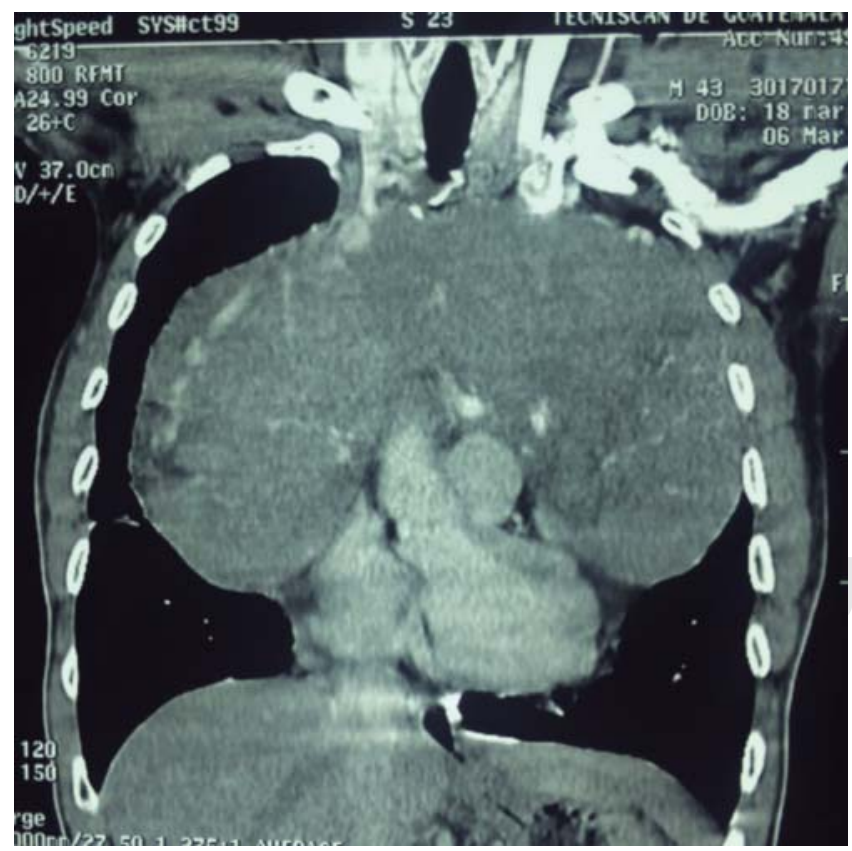

Figura 3. Timoma gigante.
Tabla 2. Características generales, $\mathrm{n}=65$.

\begin{tabular}{|c|c|}
\hline Edad, años & $\begin{array}{l}\text { 45+(IQR 24-59) Mediana } \\
\text { (rango intercuartil) }\end{array}$ \\
\hline Género, masculino & $39(60 \%)$ \\
\hline $\begin{array}{l}\text { Casos total de masas/ } \\
\text { total de casos de } \\
\text { cirugía de tórax }\end{array}$ & $65 / 844$ \\
\hline Localización & Mediastino anterior, 41 (63.07\%) \\
\hline anatómica & $\begin{array}{l}\text { Mediastino medio, } 10(15.38 \%) \\
\text { Mediastino posterior, } 14(21.53 \%)\end{array}$ \\
\hline $\begin{array}{l}\text { Patologías más } \\
\text { frecuentes }\end{array}$ & $\begin{array}{l}\text { Tumores de células germinales, } 10 \\
(15.38 \%) \\
\text { Linfomas, } 9(13.84 \%) \\
\text { Schwannoma, } 8(12.30 \%) \\
\text { Tumores del timo, } 7(10.76 \%) \\
\text { Carcinoma neuroendocrino, } \\
3(4.61 \%)\end{array}$ \\
\hline $\begin{array}{l}\text { Procedimientos } \\
\text { quirúrgicos } \\
\text { principalmente } \\
\text { realizados }\end{array}$ & $\begin{array}{l}\text { Toracotomía exploratoria, } 33 \\
(50.76 \%) \\
\text { Mediastinostomía, } 10(15.38 \%) \\
\text { Videotoracoscopia, } 7 \text { (10.76\%) } \\
\text { Biopsia ganglionar, } 5 \text { (7.69\%) }\end{array}$ \\
\hline
\end{tabular}

acuerdo con su ubicación anatómica. Las masas mediastinales benignas o malignas derivan de estructuras que normalmente se encuentran en el mediastino o que lo atraviesan durante el desarrollo, así como de las metástasis de tumores malignos que surgen en otras partes del cuerpo. Una combinación de factores clínicos y características de proyección de imagen a menudo pueden estrechar el diagnóstico diferencial cuando se detecta una masa mediastínica. En algunos casos, las características clínicas y de imagen pueden ser suficiente para guiar la terapia que a menudo incluye la resección quirúrgica que proporciona un diagnóstico definitivo. ${ }^{5}$ La literatura reporta que en el adulto $54 \%$ de los tumores mediastinales se desarrollan en el mediastino anterior, $20 \%$ en el mediastino medio y $26 \%$ en el mediastino posterior. ${ }^{2}$ En nuestra serie se mantiene la correlación por compartimentos anatómicos con pequeñas diferencias porcentuales (63, 15 y $22 \%)$. El sexo masculino predominó con el $60 \%$ de los casos.

Cuando evaluamos la frecuencia de patologías por compartimentos encontramos que en el mediastino anterior en nuestro estudio, hay coincidencia con los diagnósticos reportados por la literatura mundial, no así en la incidencia. En nuestra serie el linfoma fue el diagnóstico más frecuente y lo encontramos en el $20 \%$, teratomas en $17 \%$ y timomas en $15 \%$. No se obtuvo diagnóstico histopatológico en 4 pacientes, reportados únicamente como inflamación inespecífica crónica en 2 casos obtenidos por VATS, en uno de ellos con resultado de alfafetoproteína (AFP) en $1227 \mathrm{IU} / \mathrm{mL}$. En los 

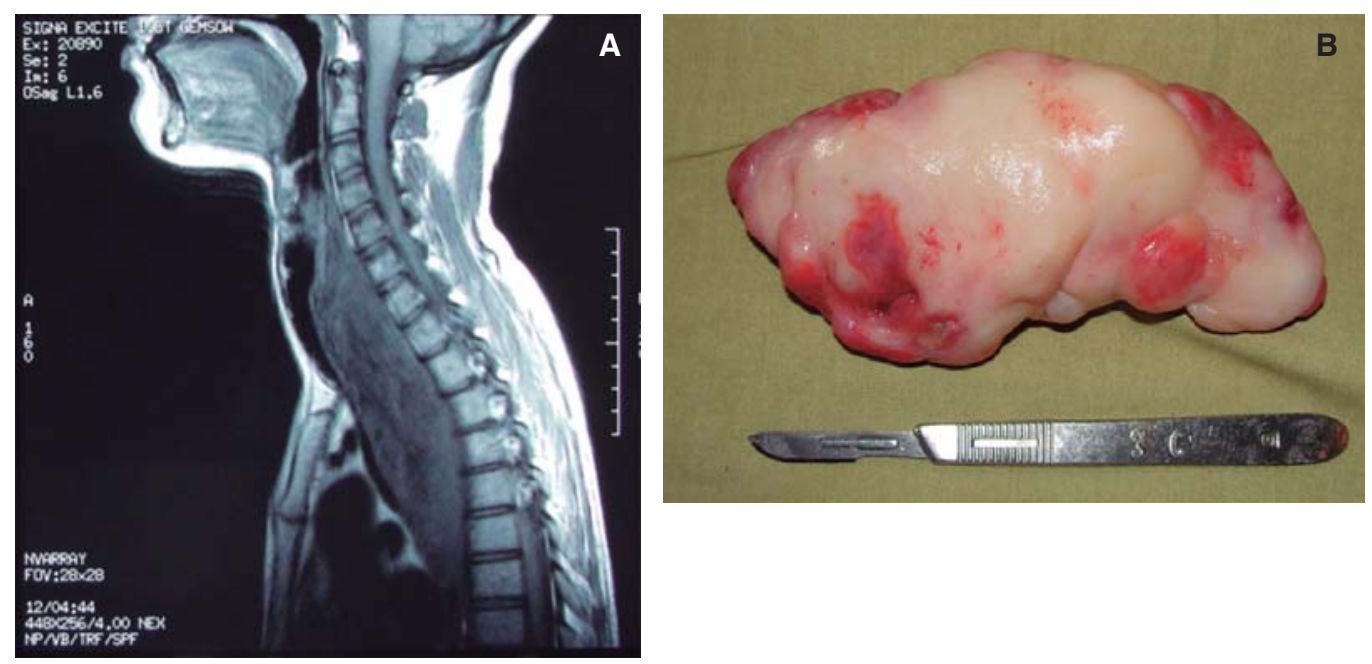

Figura 4.

Pólipo fibrovascular del esófago. A) RNM muestra tumoración intraluminal que ocupa el tercio superior y medio. B) Pieza quirúrgica de $16 \mathrm{~cm}$ de longitud.

Tabla 3. Diagnósticos por localización anatómica.

\begin{tabular}{|c|c|c|c|}
\hline & $\begin{array}{c}\text { Anterior } \\
\mathrm{n}=41(63 \%)\end{array}$ & $\begin{array}{c}\text { Medio } \\
\mathrm{n}=10(15 \%)\end{array}$ & $\begin{array}{c}\text { Posterior } \\
n=14(22 \%)\end{array}$ \\
\hline Edad, años & 40.73 & 56.60 & 46 \\
\hline \multicolumn{4}{|l|}{ Género } \\
\hline Masculino & 31 & 4 & 4 \\
\hline Femenino & 10 & 6 & 10 \\
\hline \multirow[t]{7}{*}{ Diagnósticos } & Linfoma, 8 (19.51\%) & Quiste pericárdico, 2 (20\%) & Schwannoma, 8 (57.14\%) \\
\hline & Teratoma, 7 (17.07\%) & Carcinoma neuroendocrino, 1 & Sarcoma, $1(7.14 \%)$ \\
\hline & Timoma, 6 (14.63\%) & $(10 \%)$ & Quiste broncogénico, 1 (7.14\%) \\
\hline & No diagnóstico, 4 (9.75\%) & Linfoma, 1 (10\%) & Pólipo fibrovascular, 1 (7.14\%) \\
\hline & Otros, $16(39.02 \%)$ & Adenocarcinoma & Otros, $3(14.28 \%)$ \\
\hline & & Cél. grandes 1 (10\%) & \\
\hline & & Otros, $5(50 \%)$ & \\
\hline \multirow[t]{5}{*}{ Procedimientos } & $\begin{array}{l}\text { Toracotomía exploratoria, } 14 \\
(34.14 \%)\end{array}$ & $\begin{array}{l}\text { Toracotomía exploratoria, } 5 \\
(50 \%)\end{array}$ & $\begin{array}{l}\text { Toracotomía exploratoria, } 14 \\
(100 \%)\end{array}$ \\
\hline & Mediastinostomía, 10 (24.39\%) & Ninguno, 2 (20\%) & \\
\hline & Videotoracoscopia, 6 (14.63\%) & Videotoracoscopia, 1(10\%) & \\
\hline & Biopsia ganglionar, 4 (9.75\%) & Biopsia pulmonar, 1 (10\%) & \\
\hline & Ninguno, $3(7.31 \%)$ & Broncoscopia, 1 (10\%) & \\
\hline
\end{tabular}

otros dos casos el diagnóstico fue hecho por imagen radiológica de masa mediastinal en pacientes que no regresaron a consulta o pidieron egreso contraindicado, no lográndose su confirmación. Los tumores más frecuentes del mediastino anterior son los comprendidos en las " 4 " $T$ ", timoma, teratoma, terrible linfoma y tiroides». ${ }^{5}$ El timoma es el tumor más común, afecta mayormente a los adultos con edad media de 50 años, sin predilección de sexo; más del $40 \%$ presentan síndromes paraneoplásicos tales como miastenia gravis $(30 \%)$ de todos los timomas. El teratoma es casi exclusivamente del mediastino anterior y diagnosticado a menudo en adolescentes y adultos jóvenes. La variedad teratoma maduro es la más común de todos los tumores de células germinales con un $70 \%$ en niños y $60 \%$ en adultos, son tumores encapsulados conteniendo tejido de origen ectodérmico, mesodérmico y endodérmico. Los linfomas ocupan el $20 \%$ de todos los tumores mediastinales en el adulto y $50 \%$ en los niños, siendo el más común el linfoma de Hodgkin. ${ }^{2}$

De los 10 casos reportados en nuestra serie del mediastino medio, el quiste pericárdico se observó en 2 pacientes, seguidos de linfoma y patología metastásica por adenocarcinoma. En un caso se hizo diagnóstico de adenopatía calcificada con lesión reportada por tomografía axial computarizada (TAC) de 600 unidades 
Hounsfield. La literatura reporta que en el mediastino medio las linfadenopatías son las más frecuentes y pueden ser secundarias a linfoma, sarcoidiosis o cáncer pulmonar metastásico. ${ }^{5}$ Los quistes incluyen los enterogénicos, broncogénicos y pericárdicos. Otras lesiones tales como paragangliomas, hemangiomas, linfagiomas y adenomas paratiroideos han sido descritas ocasionalmente. ${ }^{2}$

Del total de los 14 pacientes con diagnóstico de masa del mediastino posterior que atendimos, 8 (57\%) correspondieron a Schwannoma (7 benignos y 1 maligno). Los tumores neurogénicos representan la mayoría de las masas mediastinales, ${ }^{5}$ comprenden del 15 al $20 \%$ del total y alrededor del $95 \%$ se encuentran en este compartimento, siendo del 70 al $80 \%$ etiología benigna, ${ }^{2}$ tal como en nuestra serie.

Entre los casos restantes se incluyó un paciente con pólipo fibrovascular del esófago que fue resuelto exitosamente por cirugía. El pólipo fibrovascular del esófago tiene una incidencia estimada del $0.5 \%$ de los tumores benignos del esófago, alcanzando tamaño máximo reportado de $25 \mathrm{~cm}$; siendo más frecuente en hombres de 60 a 70 años. ${ }^{6}$ Nuestra paciente contaba con 19 años de edad y el pólipo resecado midió $16 \mathrm{~cm}{ }^{7}$

Los pacientes con masa mediastinal pueden presentarse con una variedad de síntomas o bien cursar asintomáticos. El común denominador siempre es el hallazgo por imagen de la presencia de una lesión en el mediastino. ${ }^{8,9} \mathrm{Si}$ los síntomas están presentes pueden ser debido al efecto directo de la masa mediastinal e incluye, tos, estridor, hemoptisis, disnea, dolor, hinchazón de extremidad superior por síndrome de cava superior, hipotensión debida a taponamiento cardíaco y síndrome de Horner; o por efecto sistémico de la enfermedad como fiebre, sudoración nocturna y pérdida de peso. ${ }^{5-8}$ La mayor parte de los pacientes atendidos consultaron por tos como síntoma único o acompañado de dolor o disnea en el $44.6 \%$. Muchos de ellos fueron referidos por otros colegas que en el transcurso de su consulta encontraron como hallazgo incidental una masa o ensanchamiento en el mediastino en el $36.92 \%$. Encontramos que aún en casos de masas extremadamente grandes como la del timoma gigante (figura 3) que ocupaba casi la totalidad de ambos hemitórax, el motivo de consulta fue disnea moderada después de correr $5 \mathrm{~km}$ cuando su meta diaria eran $10 \mathrm{~km}$. Sin embargo, los síntomas más frecuentes se pueden observar con dolor del pecho en $38 \%$, disnea en $24.6 \%$, tos en $23.9 \%$ y alrededor del $30 \%$ tienen síntomas sistémicos. ${ }^{8}$

Dado que un alto porcentaje de los pacientes son asintomáticos y el diagnóstico es sospechado por ensanchamiento mediastinal como hallazgo radiológico, la radiografía de tórax y la TAC fue obtenida en el 100\% de nuestros pacientes. No obstante, el diagnóstico de masas mediastinales requiere de múltiples métodos en los que se citan: la radiología de tórax, la cual debe distinguir masas de parénquima pulmonar. A diferencia de las masas pulmonares, las masas mediastínicas tienen ángulos obtusos de interfaz, falta de broncogramas aéreos y puede interferir con márgenes del mediastino. ${ }^{10}$

La TAC helicoidal es la herramienta más importante en la evaluación y su caracterización se basa en la atenuación específica de aire, grasa, agua y calcio.. ${ }^{11}$ Puede confirmar la presencia de masas, brindar información de anormalidades mediastinales incluyendo tamaño, localización, relación con otras estructuras, características del tejido y presencia de calcificaciones, líquido o grasa. ${ }^{5}$

Aunque la resonancia magnética nuclear puede ser muy útil en la compresión y delimitación frente a invasión del canal espinal, ${ }^{5}$ no se realizó en nuestro estudio por razones de costos. Sólo en algunos casos seleccionados y con posibilidades de costear los estudios se realizaron pruebas de marcadores tumorales para apoyar al diagnóstico en casos de masas mediastínicas anteriores bajo sospecha de timoma o tumor de células germinales. Los receptores de anticuerpos de antiacetilcolina pueden encontrarse en algunos pacientes con tumores tímicos e indican o anuncian la presencia de miastenia gravis. Niveles elevados de AFP o fracción beta de gonadotropina coriónica (beta-HCG) se asocian a tumores de células germinales. ${ }^{5}$

La broncoscopia fue utilizada en 2 pacientes (3\%) con lesiones intraluminales de neoplasias infiltrativas con biopsias endobronquiales directas. No contamos con ultrasonido (EBUS-TBNA) o aspiración con aguja fina con ecografía transesofágica (EUS-FNA) procedimientos ideales en la estadificación mediastínica.12

La utilidad y la seguridad de la biopsia transtorácica guiada por ultrasonido se ha enfatizado cada vez más al ser un fácil procedimiento realizable y seguro; ${ }^{13}$ sin embargo, por carencias del ultrasonido nuestras punciones fueron realizadas únicamente en lesiones que se encontraban en contacto a la pared torácica y guidas por la imagen de la radiografía y la tomografía de tórax. La combinación de TCNB (del inglés tru-cut needle biopsy) guiada por US o CT debe ser el primer paso en el diagnóstico tisular de las masas mediastinales. Las lesiones que no son accesibles por este método deben ser diagnosticadas por mediastinoscopia, mediastinostomía anterior o por VATS..$^{14}$ Por lo tanto, en la estrategia del diagnóstico, la muestra citohistopatológica deberá confirmar el diagnóstico presuntivo dado de acuerdo a la historia clínica y los hallazgos de estudios de gabinete obtenidos. El diagnóstico finalmente debe obtenerse del tejido afectado 
sea por resección completa del tumor, por biopsia incisional abierta o videotoracoscópica.

Las opciones estratégicas tanto terapéuticas como diagnósticas para abordar el mediastino son tan variadas como la diversidad de patologías que lo afectan y dependerán de la experiencia del cirujano y de su equipo, de la localización anatómica, de la etiología de la enfermedad, de los recursos con que se cuenta y de las comorbilidades que le acompañan. En nuestra experiencia hemos realizado en el $51 \%$ de los casos la toracotomía exploratoria posterolateral con sus grandes ventajas de excelente exposición, permitiendo siempre la toma de tejido, facilita un abordaje único para lesiones muy grandes y un mejor control de márgenes; en este procedimiento hemos abarcado todos los gestos quirúrgicos utilizados para la resección de masas localizadas en cualquiera de los compartimientos, con óptimo acceso y con mínima morbilidad. En ningún caso tuvimos que recurrir a la esternotomía media que está bien descrita para el diagnóstico y resección completa de masas mediastinales residuales después de quimioterapia por linfoma, tumores tímicos resecables, tumores de células germinales y tumores del mediastino posterior no resecables por VATS. ${ }^{2}$ La mediastinostomía anterior descrita por McNeil Chamberlain, la efectuamos en el $15 \%$ de todos los procedimientos en masas del mediastino anterior que estaban localizadas adyacentes a la pared costal en cualquiera de los hemitórax, a través de incisiones en el $3^{\circ}$ o $4^{\circ}$ espacio intercostal paraesternal con el objetivo de la toma directa de tejido. La ventaja de esta técnica es que provee material representativo y la efectividad del diagnóstico es del $90 \% .^{2}$ La VATS, la empleamos en el $10.71 \%$ para el diagnóstico y evolución de lesiones del mediastino anterior y medio, no efectuamos ninguna resección a través de ella. La VATS permite excelente exposición y disección precisa, esta técnica es de gran valor en los casos de masas con acceso difícil y que requieran visión directa tales como tumores en proximidad a estructuras neurovasculares, vasculares o del corazón; ${ }^{2}$ permite una biopsia segura de tejido, incluso cuando éste estuviera adyacente a estructuras tales como la aorta o grandes vasos. ${ }^{5}$ Desde hace más de una década se escriben las ventajas y resecabilidad de lesiones por esta vía considerándola como una técnica segura para tumores benignos mediastinales, ${ }^{15}$ así como el papel terapéutico de la cirugía video toracoscópica, siendo más significativo en el mediastino posterior, en el que se extirpó el $54 \%$ de las lesiones. ${ }^{16}$

Otro método con que se cuenta es la mediastonoscopia descrita por Carlens, útil en el diagnóstico de lesiones del mediastino anterosuperior y medio, y el abordaje transcervical como técnica invasiva que puede ser usada para realizar timectomía. ${ }^{2}$ La aspiración por broncoscopia para descomprimir lesiones quísticas agudas en pacientes no candidatos a cirugía está indicada a pesar del riesgo de introducir infección en el espacio mediastinal. ${ }^{17}$ Las punciones transtorácicas idealmente guiadas por ultrasonido o TAC, o en su defecto por una correlación clínico-radiológico de masas de gran tamaño, en relación íntima con la pared costal, practicadas a ciegas permiten la obtención de material adecuado en el $89 \%$ de los casos cuando se toman 20 más fragmentos llegando a tener una sensibilidad del $92 \%$, nosotros la hemos realizado en el $4.6 \%$ de todos los casos. Por último, la biopsia de ganglios cervical debe tenerse como una opción de mínima invasión cuando éste sea accesible en patologías en que se sospeche lesión linfoproliferativa u otro tipo de malignidad.

Concluimos que el manejo de las masas mediastinales continúa siendo un reto para el paciente que lo padece como para el equipo quirúrgico que lo trata por la diversidad de su patología, así como la complejidad de su tratamiento. Representó el $7 \%$ de los procedimientos quirúrgicos torácicos realizados. El mediastino anterosuperior fue el compartimento más afectado prevaleciendo el diagnóstico de teratoma, y la toracotomía exploratoria con resección de la masa predominó sobre las otras opciones quirúrgicas.

\section{Agradecimiento}

Dra. María Lorena Aguilera Arévalo por su valioso apoyo y revisión del artículo.

\section{REFERENCIAS}

1. Duwe BV, Sterman DH, Musani Al. Tumors of the mediastinum. Chest 2005;128(4):2893-2909.

2. Pearson's thoracic \& esophageal surgery. In: Lardinois D, Weder W, editors. Diagnostic strategies in mediastinal mass. Churchill Livingstone: Elsevier; 2008.p.15061520.

3. Shields TW. General thoracic surgery. In: Shields TW, editor. Primary lesion of the mediastinum and their investigation and treatment.4th ed. Baltimore, USA: Willians \& Wilkins; 1994. p. 3-15.

4. Pearson's thoracic \& esophageal surgery. In: Warren WH, editor. Anatomy of the mediastinum with special reference to surgical access. Churchill Livingstone: Elsevier; 2008.p.1471-1476.

5. Berry MF, Jett JR, Muller NL, Vora SR. Evaluation of mediastinal masses. Literature review current through: Sept 2015. This topic last updated: Sep 09, 2013. Access date: 2016 February 7. Available from: https://www. uptodate.com/.../approach-to-the-adult-patient-witha-Mediastinal-Masses 
6. Carreros JA, González-.Asanza C, Vaquero J, et al. Pólipo fibrovascular esofágico: a propósito de 2 casos. Gastroenterol Hepatol 2000;23(9).

7. Torres-Rodríguez T, Herrera-Cruz D, Morán OE, et al. Pólipo gigante fibrovascular del esófago. Presentación de caso único. Rev Latinoam Cir 2011;1(1):53-58.

8. Santillan-Doherty P. Tumores mediastinales. Rev Invest Clin 2006;58(3):245-253.

9. Lares C, Leite F, Bernardo J, Antunes M. Mediastinal masses: Clinical, radiological and pathological analysis of six years in a cardiothoracic surgery department. 2013 European Respiratory Society. Annual Congress. Abstrac number: 942. Publication number: p. 2943.

10. Waghray A, Sherpa L, Carpio G, Barreiro TJ. Mediastinal mass in a 25-year-old man. Chest 2014;146(2):e47-51. doi: 10.1378/chest.13-1444.

11. Juanpere S, Cañete N, Ortuño P, Martínez S, Sánchez G, Bernardo L. A diagnostic approach to the mediastinal masses. Insights Imaging 2013;4(1):29-52. doi: 10.1007/ s13244-012-0201-0.

12. Herth FJ, Rabe KF, Gasparini S, Annema JT. Transbronchial and transoesophageal (ultrasound-guided) needle aspirations for the analysis of mediastinal lesions. Eur Respir J 2006;28(6):1264-1275. doi: $10.1183 / 09031936.00013806$.
13. Yamaji $Y$, Tobino $K$, Asaji $M$, et al. The utility and safety evaluation of ultrasonography guided transthoracic biopsy. Eur Respir J 2014;44:667.

14. Petrov R, Yamakova Y, Petkova E, Petrov D, Yankov D. Minimally invasive diagnostic techniques for mediastinal lesions. European Respiratory Societ. 2012. Annual Congress. AbstracT number 4056. Publication number P3592.

15. Cheng YJ, Wu HH, Chou SH, Kao EL. Video-assisted thoracoscopic management of mediastinal tumor. JSLS 2001;5(3):241-244.

16. Pun YW, Moreno-Balsalobre R, Prieto VJ, Fernández-Fau L. Experiencia multicéntrica de cirugía Videotoracoscópica en el tratamiento de quistes y tumores del mediastino. Archivos de Bronconeumol 2002;38(9):410-414.

17. Grosu HB, Morice RC, Ost D, Eapen GA, Jimenez CA. A watery mediastinal mass. Am J Respir Crit Care Med 2013;187(10):1135-1136. doi: 10.1164/rccm.2012061055IM.

\section{$\triangle$ Correspondencia:}

Dr. Tulio Torres-Rodríguez, cirujano torácico.

Hospital San Vicente, Guatemala.

Correo electrónico: stuliotr@gmail.com

Los autores declaran no tener conflicto de intereses. 